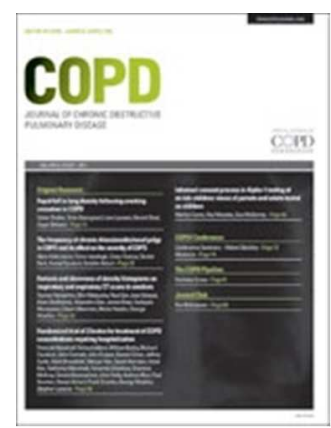

\title{
TLR4 up-regulation and reduced Foxp3 expression in mechanically ventilated smokers with obstructive chronic bronchitis.
}

\begin{tabular}{|r|l|}
\hline Journal: & COPD: Journal Of Chronic Obstructive Pulmonary Disease \\
\hline Manuscript ID: & COPD-2012-0075.R1 \\
\hline Manuscript Type: & Original Paper \\
\hline Date Submitted by the Author: & $28-J u n-2012$ \\
\hline Complete List of Authors: & $\begin{array}{l}\text { Pace, Elisabetta; Consiglio Nazionale delle Ricerche, Istituto di Biomedicina } \\
\text { e Immunologia Molecolare } \\
\text { Ferraro, Maria; Consiglio Nazionale delle Ricerche, Istituto di Biomedicina e } \\
\text { Immunologia Molecolare } \\
\text { Giarratano, Antonino; Università degli Studi di Palermo, DARE } \\
\text { Cipollina, Chiara; Fondazione Ri.Med, } \\
\text { Gjomarkaj, Mark; Consiglio Nazionale delle Ricerche, Istituto di } \\
\text { Biomedicina e Immunologia Molecolare }\end{array}$ \\
\hline Keywords: & toll like receptors, Foxp3, chemokines \\
\hline \multicolumn{2}{|c}{} \\
\hline
\end{tabular}

\section{SCHOLARONE ${ }^{\text {m }}$}

Manuscripts 
1 TITLE: TLR4 up-regulation and reduced Foxp3 expression in mechanically ventilated

2 smokers with obstructive chronic bronchitis.

3

4

5

AUTHORS: Elisabetta Pace $\mathrm{MD}^{1 *}$, Maria Ferraro $\mathrm{PhD}^{1 *}$, Antonino Giarratano $\mathrm{MD}^{2}$, Chiara

Cipollina $\mathrm{PhD}^{1-3}$, Mark Gjomarkaj MD ${ }^{1}$

8 *Elisabetta Pace and Maria Ferraro equally contributed to the manuscript.

9 FROM: ${ }^{1}$ Istituto di Biomedicina e Immunologia Molecolare (IBIM) - Consiglio Nazionale delle

10 Ricerche (CNR) - Palermo - ITALY.

112 Dipartimento di Anestesiologia, Rianimazione e delle Emergenze (DARE) - Università degli

12 Studi di Palermo - Palermo - ITALY.

133 RiMed Foundation - Palermo - Italy

14 Funded by Italian National Research Council

15 ADDRESS FOR CORRESPONDENCE: Elisabetta Pace M.D. - Istituto di Biomedicina e

16 Immunologia Molecolare -Consiglio Nazionale delle Ricerche - Via Ugo La Malfa, 153 - 90146

17 Palermo - ITALY.

18 Phone: +39.091.680-9148;

19 Fax: +39.091. 680-9122;

20 e-mail: pace@ibim.cnr.it

24 KEY WORDS: toll like receptors, Foxp3, chemokines, smokers, respiratory failure.

RUNNING HEAD: Innate immunity and immune regulation in acute respiratory failure. 


\section{LIST OF ABBREVIATIONS}

$27 \mathrm{COPD}=\mathrm{Chronic}$ obstructive pulmonary disease $\mathrm{C}=$ Controls; $\mathrm{CB}=$ chronic bronchitis; $\mathrm{S}=$ smokers;

28 Mini-BAL= mini-bronchoalveolar lavage; TLR= Toll like receptor; Foxp3=f forkhead box P3; IP-

$2910=$ interferon gamma induced protein $10 ;$ IL-8= interleukin 8.

30 
32 Elisabetta Pace- Competing interests: None declared.

33 Maria Ferraro - Competing interests: None declared.

34 Antonino Giarratano- Competing interests: None declared.

35 Chiara Cipollina-Competing interests: None declared.

36 Mark Gjomarkaj- Competing interests: None declared. 


\section{SUMMARY}

41 Background. Chronic bronchitis (CB) is a risk factor in chronic obstructive pulmonary disease 42 (COPD) for accelerated lung function decline and increased mortality. The lung and systemic 43 inflammatory and immunological profile of COPD patients with CB which acutely experience 44 respiratory failure upon a disease exacerbation is unknown.

45 Methods. In this study, we explored the expression of Foxp3 by western blot analysis, TLR4 by 46 immunocytochemistry and the concentrations of IP-10 and IL-8 by ELISA in the mini-

47 bronchoalveolar lavages (mini-BAL) and in the peripheral blood of patients with respiratory failure 48 requiring intubation and mechanical ventilation. The recruited subjects were separated into three 49 different groups: smokers with CB and COPD (COPD, $n=18$ ), smokers with CB but without COPD $50(\mathrm{~S}, \mathrm{n}=8)$ and patients without $\mathrm{CB}$ and without $\mathrm{COPD}(\mathrm{C}, \mathrm{n}=10)$.

51 Results. In mini-BAL of COPD group, Foxp3 and IP-10 were significantly reduced while TLR4 52 was significantly increased in comparison to C. TLR4 was also increased in mini-BAL of S. In 53 COPD peripheral blood, Foxp3 was reduced in comparison to $\mathrm{C}$ but no significant differences were 54 observed for TLR4 and for IP-10. No significant differences were observed for IL-8 concentrations 55 in the mini-BAL and in the blood of the recruited patients. The mini-BAL TLR4 expression 56 correlated with the Clinical Infective Pulmonary Score.

57 Conclusions. In exacerbated COPD patients with respiratory failure, lung and systemic reduced 58 immune regulatory events (low Foxp3 expression) and lung increased innate immunity responses 59 (high TLR4 expression) occur. These events may contribute to the increased inflammatory events 60 leading to respiratory failure. 
62

63

64

65

66

67

68

69

70

71

72

73

74

75

76

77

78

79

80

81

82

83

84

85

86

\section{INTRODUCTION}

Chronic obstructive pulmonary disease (COPD) is a heterogeneous disease, is associated with pulmonary and extra-pulmonary clinical manifestations and includes different clinically relevant subtypes (1). One of the COPD subtypes is characterized by chronic bronchitis (CB). CB is a risk factor for accelerated lung function decline in COPD, increased hospitalization, and increased mortality (2).

Cigarette smoke represents the most important risk factor for $\mathrm{CB}$ (3). Chronic oxidative stress of cigarette smoking induces mucus secretion and the increased mucus viscosity renders the airways susceptible to bacterial infections, a hallmark of CB (3).

A key component of the innate immunity and of the innate defence mechanisms against infections is represented by the toll like receptor (TLR) family (4) .

A recent hypothesis regarding COPD pathogenesis suggests as "step 1 of the disease" the activation of innate responses by injured tissue components (5). Products derived from epithelial cell injury can act as ligands for TLR4 and TLR2, thus amplifying inflammatory responses within the airways. Cigarette smoke is able to increase the expression of TLR4 and to orientate the activation of TLR4 toward an increased release of IL-8 and a reduced release of interferon gamma-induced protein 10 (IP-10) in bronchial epithelial cells (6).

In the airways of COPD patients mechanically ventilated due to acute respiratory failure, there is an increased expression of TLR4 and an increased chemotactic activity toward neutrophils but a reduced concentration of IP-10 with a reduced chemotactic activity toward lymphocytes (7). The increased airway inflammation in the airways of COPD patients may also be sustained by the impairment of immune regulatory events and in particular may be linked to the reduced expression of the forkhead box P3 (Foxp3), a transcription factor crucially involved in T regulatory activities. COPD patients have, in small airways, decreased numbers of Foxp3 positive cells that negatively correlate with airflow obstruction (8) (9). Although COPD is associated with lung and systemic 
87 inflammation, it is unknown whether the alteration in the innate and immune regulatory events

88 observed in the airways of COPD patients are also present in the systemic compartment.

89 The objectives of this study were to investigate the immune regulatory events and the inflammatory

90 and the host defence responses in the airways and in the systemic compartment of COPD patients

91 with CB. Exacerbated COPD patients who experienced an acute respiratory failure requiring

92 endotracheal intubation and mechanical ventilation were compared to patients requiring also

93 intubation and mechanical ventilation but without COPD and without $\mathrm{CB}$. In the recruited patients

94 the expression of TLR4, the expression of Foxp3 and the release of specific chemokines (IP-10 and

95 IL-8) were investigated. 


\section{MATERIALS AND METHODS}

98

99

100

101

102

103

104

105

106

107

108

109

110

111

112

113

114

115

116

117

118

119

120

121

\section{Patient population}

This study was conducted at the ICU of the Department of Anestesiology, Reanimation and Emergency of the University of Palermo, Italy. Local ethic committee permission and informed written consent from either the patient or closest relatives were obtained. The patients were classified into the following groups: 1) subjects without smoking history, without history of previous $\mathrm{CB}$ or chronic pulmonary diseases including asthma and COPD and with acute respiratory failure upon surgery for abdominal or thoracic aneurysm, (Controls $=\mathrm{C} ; \mathrm{n}=10) ; 2$ ) smoking subjects (>15 pack-year) with $\mathrm{CB}$, without $\mathrm{COPD}$ and with acute respiratory failure upon surgery for abdominal or thoracic aneurysm $(\mathrm{S} ; \mathrm{n}=8) ; 3)$ smoking subjects ( $>15$ pack-year) with $\mathrm{CB}$ with COPD (GOLD-1-2) and with acute respiratory failure upon treatment failure of an acute exacerbation (COPD; $n=18$ ). The COPD patients fulfilled the diagnostic criteria of COPD (10) with a post-bronchodilator obstruction $\left(\mathrm{FEV}_{1}<80 \%\right.$ predicted, and $\mathrm{FEV}_{1} / \mathrm{FVC}$ ratio $\left.<70 \%\right)$. Patients with x-ray or clinical evidence of sepsis or pneumonia at the time of mini-bronchoalveolar lavage (mini-BAL) collection were not included. CB were defined on the basis of symptoms and in particular productive cough lasting more than three months in more than two years. All recruited subjects required mechanical ventilation and underwent therapy with antibiotics and systemic corticosteroids (no significantly different doses among the patients included in the three groups).

The antibiotics were adjusted to cover any identified pathogens on the basis of antibiograms. COPD patients, before COPD exacerbation, were undergoing therapy with bronchodilators but not with corticosteroids. Exacerbations were defined as previously reported (10) and were treated with antibiotics and systemic corticosteroids. At the ICU admission, data for Clinical Pulmonary Infective Score (CPIS), the simplified Acute Physiology Score (SAPS II) and sepsis-related organ failure assessment (SOFA) were collected from each recruited patient. Paired mini-BAL and blood samples were collected from all participants. Microbiology of mini-BAL was also assessed. 
122 mini-BAL collection and processing

123 Distal lung fluid samples (mini-BAL) were obtained using BAL Cath system (by Kimberly Clark)

124 within $1 \mathrm{~h}$ from the intubation. The protected catheter was blindly advanced through the

125 endotracheal tube until it was wedged into a distal airway and aliquots of $10 \mathrm{ml}$ of sterile $0.9 \%$

$126 \mathrm{NaCl}$ were instilled and gently suctioned (recovered volume about $70 \%$ of the instilled volume).

127 Mini-BAL samples were filtered through a sterile gauze and then centrifuged at $1300 \mathrm{rpm}$ for 10

128 min to separate cells from supernatants. Total and differential (diff-quick staining) cell counts were

129 assessed. The cell fraction was used for immunocytochemistry and western blot experiments. The

130 supernatants were assessed for cytokine levels.

\section{Blood samples}

132 Blood samples $(10 \mathrm{ml})$ were collected from C, S, COPD subjects and then processed for obtaining

133 plasma and peripheral blood mononuclear cells (PBMC). PBMC were isolated from blood s by

134 Ficoll-Hypaque (Pharmacia) gradient centrifugation. The cells were suspended in RPMI 1640 tissue

135 culture medium (Invitrogen Life Technologies) supplemented with 1\% heat-inactivated FCS

136 (Invitrogen Life Technologies), $2 \mathrm{mM}$ L-glutamine, $20 \mathrm{mM}$ HEPES, $100 \mathrm{U} / \mathrm{ml}$ penicillin, $100 \mu \mathrm{g} / \mathrm{ml}$

137 streptomycin, $5 \times 10^{-5} \mathrm{M} 2-\mathrm{ME}$ and $85 \mu \mathrm{g} / \mathrm{ml}$ gentamicin. Purity and viability were tested using

138 trypan blue exclusion.

139 Immunocytochemistry

140 The expression of TLR4 was evaluated using a rabbit polyclonal antibody (Santa Cruz

141 Biotechnology). Immunocytochemistry was performed using AP-LSAB2 (DAKO, Glostrup,

142 Denmark) kit following the manufacturer's instructions and new Fuchsin as chromogenic substrate

143 (DAKO) (cytoplasmic red staining). Negative controls were performed using rabbit or mouse

144 negative control immunoglobulins (DAKO). Data are expressed as percentage of positive cells.

145 Western blot analysis 
146 The expression of Foxp3 was evaluated by western blot analysis as previously described (11) with

147 minor modifications. $40 \mu \mathrm{g}$ of total protein were loaded in the gel. All blots were probed using a

148 goat polyclonal antibody anti-Foxp3 (1:100) (Santa Cruz Biotechnology, Santa Cruz, CA).

149 Membranes were then stripped and incubated with goat polyclonal anti- $\beta$-actin (Sigma) as

150 housekeeping protein to normalize differences in protein loading. Revelation was performed with an

151 enhanced chemioluminescence system (GE Healthcare, Chalfont St. Giles, UK) followed by

152 autoradiography. Negative controls were performed in the absence of primary antibody or including

153 an isotype control antibody. Data are expressed as densitometric arbitrary units by correction with

154 the density of the bands obtained for beta-actin.

\section{Measurement of IL-8 and IP-10}

156 The concentrations of IL-8 and IP-10 in plasma from C, S, CB-COPD subjects were determined by

157 an enzyme-linked immunosorbent assays (ELISA) following the manufacturer's instructions

158 (Quantikine; R\&D Systems, Minneapolis, MN).

\section{Statistics}

160 Data are expressed as median (25-75 percentiles). Kruskal Wallis test was performed for

161 comparisons among patient groups. A non-parametric Mann Whitney test was then applied as the

162 initial Kruskal Wallis test was significant. The Wilcoxon test was used for comparisons between

163 mini-BAL and autologous peripheral blood in each recruited patient. The Spearman test was used

164 for correlations. $\mathrm{P}<0.05$ was accepted as statistically significant. 165 
RESULTS

167 Demographic characteristics of the subjects.

168 The demographic characteristics of the three study groups are shown in Table 1. SAPS II and SOFA

169 scores revealed no significant differences in the recruited patients. The CPIS score was significantly

170 higher in COPD than in C (Table 1). No significant differences for CPIS score were shown in $\mathrm{S}$ in

171 comparison to $\mathrm{C}$ and to COPD. The total and the differential cell counts of mini-BAL are shown in

172 Table 2. Significantly higher numbers of total cells and of neutrophils were present in COPD

173 patients. Microbiology of mini-BAL was shown in table 3.

174

175 Expression of TLR4 in cells from mini BAL and from peripheral blood cells.

176 The percentage of TLR4 positive cells was significantly higher in mini-BAL cells from COPD and

177 from $\mathrm{S}$ in comparison to mini-BAL from C. The percentage of TLR4 positive cells was significantly

178 higher in mini-BAL cells from COPD in comparison to mini-BAL from $\mathrm{S}$ and to autologous

179 peripheral blood (figure 1) (table 4). No significant differences in TLR4 expression were observed

180 in peripheral blood cells among C, S and COPD (figure 1) (table 4).

181 Concentrations of IL-8 and of IP-10 in mini BAL and in peripheral blood cells.

182 The concentrations of IL-8 were significantly higher in mini-BAL of all the recruited patients $(\mathrm{C}, \mathrm{S}$

183 and COPD patients) in comparison to autologous peripheral blood (figure 2). No significantly

184 different concentrations of IL-8 were observed in mini-BAL and in peripheral blood among C, S

185 and COPD (figure 2). The IP-10 concentrations were significantly reduced in mini-BAL from

186 COPD in comparison to $\mathrm{C}$ and $\mathrm{S}$ and in comparison to autologous peripheral blood (figure 3 ). The

187 IP-10 concentrations were significantly increased in peripheral blood from COPD in comparison to

188 mini-BAL from C. No significantly different concentrations of IP-10 were observed in peripheral

189 blood between S and C (figure 3). 
190 Expression of Foxp3 in mini BAL and in peripheral blood cells.

191 In COPD and in S the expression of Foxp3 in mini-BAL and in peripheral blood was significantly

192 lower than in C (figure 4 A-B) (table 4).. In COPD the expression of Foxp3 in mini-BAL and in

193 peripheral blood was significantly lower than in S (figure 4 A-B) (table 4).. No significant

194 differences were observed in the expression of Foxp3 in mini-BAL and in autologous peripheral

195 blood in all the recruited patients.

\section{Correlations}

197 Finally, we tested whether the observed alterations in TLR4, Foxp3 and IP-10 in both lung and

198 systemic compartments correlate with clinical scores of severity. CPIS correlates with mini-BAL

199 TLR4 expression (figure 5) but not with the other markers (Foxp3 and IP-10) (data not shown). No

200 significant correlations were observed between SOFA or SAPS II score with any of the tested 201 markers (data not shown). 
DISCUSSION

204 COPD is a heterogeneous disease and includes different clinically relevant subtypes. A divergent 205 distribution of parenchymal (emphysema) and bronchial airway (chronic bronchitis) (CB) disease 206 contributes to the phenotypic heterogeneity in COPD (12). COPD is associated not only with an 207 abnormal inflammatory response in the lung but also with systemic inflammation, including 208 systemic oxidative stress, activation of circulating inflammatory cells and increased circulating 209 levels of inflammatory cytokines (12). The low-grade systemic inflammation present in COPD 210 patients may be responsible for the systemic clinical manifestations of the disease including 211 malnutrition, muscle wasting, osteoporosis, cardiovascular diseases, type II diabetes, anaemia and 212 depression (13).

213 This study explored whether lung and systemic inflammation occur concurrently and similarly in 214 patients with acute respiratory failure with and without CB or COPD. We demonstrated for the first 215 time that patients with both CB and COPD who underwent an acute exacerbation have a different 216 inflammatory profile (IP-10 levels) and a different alteration in the innate immune responses (TLR4 217 expression) in the airways and in the systemic compartment. Differently, in these patients a similar 218 alteration in the immune regulatory events (low Foxp3 expression) was observed in the airways and 219 in the systemic compartment. The novel aspects underlined by the present study are related to the 220 accurate selection of patients with a similar phenotype (CB) and a similar exposure to risk factor 221 (cigarette smoke) in order to limit the variability of the obtained results and to better identify the 222 role of the observed alterations in airway obstruction. Most of the studies report data from smoker 223 or COPD patients with different phenotypes.

224 CB is defined on the basis of chronic cough and sputum due to mucus hypersecretion and 225 histologically, it is characterised by airway inflammation, hypertrophy of submucosal mucus 226 secreting glands, and goblet cell hyperplasia (14). CB is a risk factor in COPD for accelerated lung 227 function decline, increased hospitalization, and increased mortality (2). In this regard it has been 228 demonstrated that the presence of CB may compromise the sterility of distal airway supporting the 


\section{hypothesis of natural progression from the simple mucus hypersecretion to purulent hypersecretion} and obstructive bronchitis ( Hogg, Lancet 2004). Here, it is showed that the expression of TLR4 is increased in COPD patients with CB and acute exacerbation in the airways but not in the systemic compartment and this alteration is observed at lower extent also in smokers with CB but without COPD and without acute exacerbation. TLR4 expressed is associated in these patients with the presence of neutrophils which represent the predominant cell type in COPD patients. The data provided extend and integrate previous results from our group showing that in the airways of COPD patients with acute respiratory failure, there is an increased expression of TLR4 (7). Our data demonstrate that TLR4 expression is higher in mini-BAL cells in comparison to blood compartment suggesting an up-regulation of TLR4 at the transit from blood into the airway compartment. This phenomenon seems to be specific for TLR4 since it has been previously demonstrated that in COPD patients the expression of TLR2 is lower on sputum neutrophils in comparison to blood compartment indicating a down-regulation of TLR2 at the transit from blood into the airway compartment (16).

TLR4 signaling, through MyD88 and IRAK1, plays a predominant role as a regulator of smokeinduced protease production (17). Furthermore, CSE increase the expression of TLR4 but not of TLR2 and modify the functional activation of TLR4 generating an imbalance between cytokines with opposite functions such as IL-8 and IP-10 (6). IP-10 concentrations in mini-BAL of smoker COPD who are mechanically ventilated for acute exacerbation are reduced in comparison to another group of patients mechanically ventilated but not smokers and without COPD (7). We confirm here the presence of reduced concentrations of IP-10 within the airways of mechanically ventilated and exacerbated COPD patients and demonstrate for the first time that within the systemic compartment in the same patients an increased concentration of IP-10 is observed in comparison to patients with acute respiratory failure but without $\mathrm{CB}$ and COPD. Nasal epithelial cells obtained from smokers create an overall cytokine microenvironment that after infection with influenza suppresses the 
254 concentrations of IP-10 (18). When the bronchial epithelial cells were exposed to CSE, the release

255 of IP-10 decreases while the release of IL-8 increases (6). Although no significantly different

256 concentrations of IL-8 were observed in mini-BAL and in peripheral blood between COPD, S and C

257 within the airways the elevated concentrations of IL- 8 are not balanced by elevated IP-10

258 concentrations. The prevalence of IL-8 may in turn sustain the influx of neutrophils into the airways

259 thus triggering innate immunity responses, while IP-10 attracts monocytes and lymphocytes (19)

260 thus promoting activation of adaptative responses to efficiently and specifically limit microbial

261 invasion and to restrain the harmful effects of prolonged neutrophil activation. The findings that

262 mini-BAL from COPD had reduced IP-10 concentrations and reduced chemotactic activitites

263 toward lymphocytes (7) might contribute to explain why the differential cell counts of mini-BAL

264 from COPD failed to have lymphocytes and prompted us to explore whether lymphocyte regulatory

265 activities were altered in these patients. $\mathrm{CD} 4^{+} \mathrm{Foxp}^{+}$regulatory $\mathrm{T}$ lymphocytes (Treg) are a

266 subclass of $\mathrm{CD} 4^{+} \mathrm{T}$ cell receptor (TCR) $\alpha \beta^{+} \mathrm{T}$ cells that are essential to preserve immune

267 homeostasis (20), (21). Stable expression of the transcription factor Foxp3 is a prerequisite for the

268 maintenance of suppressive properties in CD4+ regulatory T cells. Foxp3 mRNA expression is not

269 itself sufficient for stable Foxp3 protein expression (22). The epigenetic modifications, such as

270 histone modification or DNA methylation, control regulatory T cells by controlling Foxp3 gene

271 expression through altering the accessibility of the Foxp3 locus and by acetylating or deacetylating

272 Foxp3 protein, thereby enabling the epigenetic regulation of Foxp3 target genes. Absence of the

273 Foxp3 transcription factor at systemic level leads to the rapid development of fulminant multiorgan

274 autoimmunity. A decreased Foxp3 expression in COPD patients and smokers parallels the

275 aggravation of the disease (23). Patients with moderate or severe COPD upon fluticasone and

276 salmeterol combination therapy show an increased proportion of Foxp3+Tregs in the total

277 peripheral blood CD4+T cell population (24). Smokers with normal lung function and COPD

278 patients have increased numbers of Foxp3-positive cells in large airways but they have decreased

279 numbers of Foxp3-positive cells in small airways (25). In the patients with acute respiratory failure 
280

recruited in the present study, the decreased expression of Foxp3 is present in smokers and in COPD in the distal airways and in the systemic compartment suggesting that the alterations in the immune regulatory activities are early events in the disease and may contribute to the systemic effects of the disease. It is conceivable that cigarette smoke may contribute to induce epigenetic modifications leading to the reduced expression of Foxp3 in smokers and in COPD smokers at both the airways and systemic compartment. No study has already specifically addressed this point yet.

Finally, the relevance of the observed alterations in the severity of the recruited patients was assessed. Several commonly used scoring systems exist assessing the severity of the disease in critically ill patients by predicting mortality. In the present study the recruited patients were classified on the basis of their SOFA, SAPS II and CPIS score. The mortality in elderly patients was higher than that of the younger patients and SAPS II (26) was an independent predictor of mortality in elderly patients with sepsis (27). The SOFA score, widely used in many cardiac surgical intensive units, is used for grading organ dysfunction or failing organ system (28) . Prognostic relevance of the SOFA score in combination with inflammatory parameters was also found in a recent study conducted by Zügel et al. (29). The CPIS score is calculated on the basis of points assigned for various signs and symptoms of pneumonia (eg, fever and extent of oxygenation impairment) and a CPIS >6 may serve as a surrogate tool to facilitate the diagnosis of ventilatorassociated pneumonia (30). In the present study, recruited patients have no significant different SOFA or SAPS II scores and these scores did not correlate with any of the tested markers. The only differences among the recruited patients were related to the CPIS score and CPIS score correlates with TLR4 mini-BAL expression. Future studies on a larger cohort of patients are needed to clarify whether the assessment of TLR4 expression in miniBAL may improve the predictive value of CPIS to early identify patients with ventilator-associated pneumonia.

In conclusion, patients with both $\mathrm{CB}$ and COPD who underwent an acute exacerbation have a different alteration in the levels of IP-10 and in TLR4 expression in the airways and in the systemic 
305 compartment while they have a similar alteration in Foxp3 expression in the airways and in the

306 systemic compartment. The inflammatory profile of the COPD patients in the present study may be

307 the result of the failure to properly clear the inflammatory cells after the exacerbation of the disease.

308 
ACKNOWLEDGEMENTS

310

311 This work was supported by the Italian National Research Council.

312 Elisabetta Pace and Maria Ferraro designed the study, performed the statistical analysis of the data e

313 wrote the manuscript and declares that they have had access to and takes responsibility for the

314 integrity of the data and the accuracy of the data analysis.

315 Chiara Cipollina performed all the experiments of the study and participated to the interpretation of 316 the data.

317 Antonino Giarratano contributed to the patient selection, collected and managed biological 318 samples.

319 Mark Gjomarkaj contributed to the interpretation of the data and to the writing out of the 320 manuscript. 


\section{REFERENCES}

326 1) Garcia-Aymerich J, Serra Pons I, Mannino DM, Maas AK, Miller DP, Davis KJ. Lung function

327 impairment, COPD hospitalisations and subsequent mortality. Thorax. 2011 Jul;66(7):585-90.

328 2) Vestbo J, Prescott E, Lange P. Association of chronic mucus hypersecretion with FEV1 decline 329 and chronic obstructive pulmonary disease morbidity. Am J Respir Crit Care Med 1996;153:15303301535.

331 3) Mullen JB, Wright JL, Wiggs BR, Pare PD, Hogg JC. Structure of central airways in current 332 smokers and ex-smokers with and without mucus hypersecretion: relationship to lung function; 333 Thorax 1987;42: 843-848.

334 4) Zhang, G. and S. Ghosh, Molecular mechanisms of NF-kappaB activation induced by bacterial 335 lipopolysaccharide through Toll-like receptors. J Endotoxin Res, 2000. 6: 453-7.

336 5) Cosio MG, Saetta M, Agusti A. Immunologic Aspects of Chronic Obstructive Pulmonary 337 Disease. N Engl J Med 2009; 360: 2445-2454.

338 6) Pace E, Ferraro M, Siena L Melis M, Montalbano A, Johnson M, Bonsignore MR, Bonsignore 339 G, Gjomarkaj M. Cigarette smoke increases TLR4 and modifies LPS mediated responses in airway 340 epithelial cells. Immunology, 2008; 124:401-11.

341 7) Pace E, Giarratano A, Ferraro M, Bruno A, Siena L, Mangione S, Johnson M, Gjomarkaj M.

342 TLR4 upregulation underpins airway neutrophilia in smokers with chronic obstructive pulmonary 343 disease and acute respiratory failure. Hum Immunol., 2011; 72: 54-62.

344 8) Isajevs S, Taivans I, Strazda G, Kopeika U, Bukovskis M, Gordjusina V, Kratovska A.

345 Decreased FOXP3 expression in small airways of smokers with COPD. Eur Respir J. 2009 346 Jan;33(1):61-7. 
347 9) Roos-Engstrand E, Pourazar J, Behndig AF, Bucht A, Blomberg A. Expansion of CD4+CD25+

348 helper T cells without regulatory function in smoking and COPD. Respir Res. 2011 Jun 8;12:74.

349 10) Pauwels RA, Buist AS, Calverley PM, Jenkins CR, Hurd SS : Global strategy for the diagnosis, 350 management, and prevention of chronic obstructive pulmonary disease. NHLBI/WHO Global 351 Initiative for Chronic Obstructive Lung Disease (GOLD) workshop summary. Am J Respir Crit 352 Care Med 2001, 163:1256-1276.

353 11) Pace E., Ferraro M., Uasuf C.G., La Grutta S., Liotta G., Giarratano A., Johnson M., 354 Gjomarkaj M. Cilomilast counteracts the effects of cigarette smoke in innate responses of airway 355 epithelial cells. Cellular Immunology 2011; 268: 47-53.

356 12) Calverley PM, Walker P. Chronic obstructive pulmonary disease. Lancet 2003;362:1053-1061.

357 13) Agustí A..Systemic effects of chronic obstructive pulmonary disease: what we know and what 358 we don't know (but should). Proc Am Thorac Soc. 2007 Oct 1;4(7):522-5.

359 14) Reid L. Pathology of chronic bronchitis. Lancet 1954;i:275-279.2. Wilson, R., Evidence of 360 bacterial infection in acute exacerbations of chronic bronchitis. Semin Respir Infect, 2000. 15: 20836115.

362 15) Hogg JC. Pathophysiology of airflow limitation in chronic obstructive pulmonary disease.

363 Lancet, 2004. 364: 709-721.

364 16) von Scheele I, Larsson K, Dahlén B, Billing B, Skedinger M, Lantz AS, Palmberg L. Toll-like 365 receptor expression in smokers with and without COPD. Respir Med. 2011;105:1222-30. 366

367 17) Geraghty P, Dabo AJ, D'Armiento J. TLR4 protein contributes to cigarette smoke-induced 368 matrix metalloproteinase-1 (MMP-1) expression in chronic obstructive pulmonary disease. J Biol 369 Chem. 2011;286: 30211-30218. 
371 18) Horvath KM, Brighton LE, Zhang W, Carson JL, Jaspers I. Epithelial cells from smokers 372 modify dendritic cell responses in the context of influenza infection. Am J Respir Cell Mol Biol. $3732011 ; 45: 237-245$.

374

375 19) Boodoo S, Spannhake EW, Powell JD, Horton MR. Differential regulation of hyaluronan376 induced IL-8 and IP-10 in airway epithelial cells. Am J Physiol Lung Cell Mol Physiol 2006; 291: 377 L479-L486

378 379 380 381 382 383 384

385 386 387

20) J.D. Fontenot, A.Y. Rudensky. A well adapted regulatory contrivance: regulatory T cell development and the forkhead family transcription factor Foxp3. Nat Immunol, 6 (2005), pp. 331337

21) S. Sakaguchi, M. Miyara, C.M. Costantino, D.A. Hafler. FOXP3+ regulatory T cells in the human immune system. Nat Rev Immunol, 10 (2010), pp. 490-500

22) S. Hori. Rethinking the molecular definition of regulatory T cells. Eur J Immunol, 38 (2008), pp. 928-930.

23) Chu S, Zhong X, Zhang J, Lao Q, He Z, Bai J. The expression of Foxp3 and ROR gamma t in lung tissues from normal smokers and chronic obstructive pulmonary disease patients. Int Immunopharmacol. 2011 Nov;11(11):1780-8. Epub 2011 Jul 23.

24) Yang L, Ma QL, Yao W, Zhang Q, Chen HP, Wang GS, Wang CZ. Relationship between the anti-inflammatory properties of salmeterol/fluticasone and the expression of CD4 $\square \mathrm{CD} 25 \square$ Foxp3 $\square$ regulatory T cells in COPD. Respir Res. 2011 Oct 28;12:142.

25) Isajevs S, Taivans I, Strazda G, Kopeika U, Bukovskis M, Gordjusina V, Kratovska A.

Decreased FOXP3 expression in small airways of smokers with COPD. Eur Respir J. 2009 Jan;33(1):61-7.

26) Le Gall Jr, Lemeshow S, Saulnier F. A new Simplified Acute Physiology Score (SAPS II) based on a European/North American multicenter study. JAMA 1993, 270: 2957-2963. 
396 27) Tiruvoipati R, Ong K, Gangopadhyay H, Arora S, Carney I, Botha J. Hypothermia predicts

397 mortality in critically ill elderly patients with sepsis. BMC Geriatr. 2010 Sep 27;10:70.

398 28) Vincent JL, de Mendonça A, Cantraine F, Moreno R, Takala J, Suter PM, Sprung CL, Colardyn

399 F, Blecher S. Use of the SOFA score to assess the incidence of organ dysfunction/failure in

400 intensive care units: results of a multicenter, prospective study. Working group on "sepsis-related

401 problems" of the European Society of Intensive Care Medicine.Crit Care Med. 1998

402 Nov;26(11):1793-800.

403 29) Zügel NP, Kox M, Lichtwark-Aschoff M, Gippner-Steppert C, Jochum M: Predictive relevance

404 of clinical scores and inflammatory parameters in secondary peritonitis. Bull Soc Sci Med Grand

405 Duche Luxemb. 2011, 1:41-71.

406

407 30) Zilberberg MD, Shorr AF. Clin Infect Dis. Ventilator-associated pneumonia: the clinical 408 pulmonary infection score as a surrogate for diagnostics and outcome. Clin Infect Dis 2010 Aug 409 1;51 Suppl 1: S131-5.

410

411

412 


\section{LEGENDS TO THE FIGURES}

414 Figure1. Increased expression of TLR4 in mini-BAL but not in the peripheral blood of S and

415 of COPD. Mini-BAL cells and paired blood samples were recovered from C $(n=10)$, from $S(n=8)$

416 and from COPD $(n=18)$ patients. The expression of TLR4 was assessed by immunocytochemistry

417 using an anti-TLR4 polyclonal antibody; ${ }^{*} \mathrm{p}<0.05$ vs $\mathrm{C} ;{ }^{* *} \mathrm{p}<0.05 \mathrm{vs} \mathrm{S} ; \# \mathrm{p}<0.05$ vs autologous

418 peripheral blood. Data are expressed as median (25-75 percentiles).

419 Figure 2. Absence of differences in the concentrations of IL-8 in mini-BAL and in the

420 peripheral blood of S and of COPD. Mini-BAL supernatants and paired blood samples were

421 recovered from $C(n=10)$, from $S(n=8)$ and from COPD $(n=18)$ patients. IL-8 concentrations were

422 measured by ELISA as described in "materials and methods" and are expressed as pg/ml. Data are

423 expressed as median (25-75 percentiles).

424 Figure 3. Reduced concentrations of IP-10 in mini-BAL but not in the peripheral blood of

425 COPD. Mini-BAL supernatants and paired blood samples were recovered from $C(n=10)$, from $S$

$426(\mathrm{n}=8)$ and from COPD $(\mathrm{n}=18)$ patients. IP-10 concentrations were measured by ELISA as

427 described in "materials and methods" and are expressed as pg/ml. * p<0.05 vs C; ${ }^{* *} \mathrm{p}<0.05$ vs S; \# $428 \mathrm{p}<0.05$ vs autologous peripheral blood. Data are expressed as median (25-75 percentiles).

429 Figure 4. Reduced expression of Foxp3 in mini-BAL and in the peripheral blood of S and of

430 COPD. Mini-BAL cells and paired blood samples were recovered from $C(n=10)$, from $S(n=8)$ and

431 from COPD $(n=18)$ patients. Total proteins were extracted and analysed for Foxp3 expression by

432 western blot analysis. Membranes were then stripped and incubated with goat polyclonal anti- $\beta$ -

433 actin.. A. Densitometric analysis of Foxp3 expression. Signals corresponding to Foxp3 on the

434 various western blots were semiquantified by densitometric scanning, normalized and expressed

435 after correction with the density of the band obtained for beta-actin (mean \pm SD). $* \mathrm{p}<0.05$. B.

436 Representative western blot analysis for Foxp3 expression from C, S and COPD subjects. Data are 437 expressed as median (25-75 percentiles). 
1

2

3

4

5

6

7

8

9

10

11

12

13

14

15

16

17

18

19

20

21

22

23

24

25

26

27

28

29

30

31

32

33

34

35

36

37

38

39

40

41

42

43

44

45

46

47

48

49

50

51

52

53

54

55

56

57

58

59

60

438 Figure 5. Correlations between the expression of TLR4 in miniBAL and CPIS score. The

439 expression of TLR4 in mini-BAL of $C(n=10), S(n=8)$ and COPD $(n=18)$ was correlated with

440 CPIS score by Spearman Correlation test. 
Table 1: Demographic and clinical characteristics of the subjects:

3

4 
Table 2: Microbiology of miniBAL

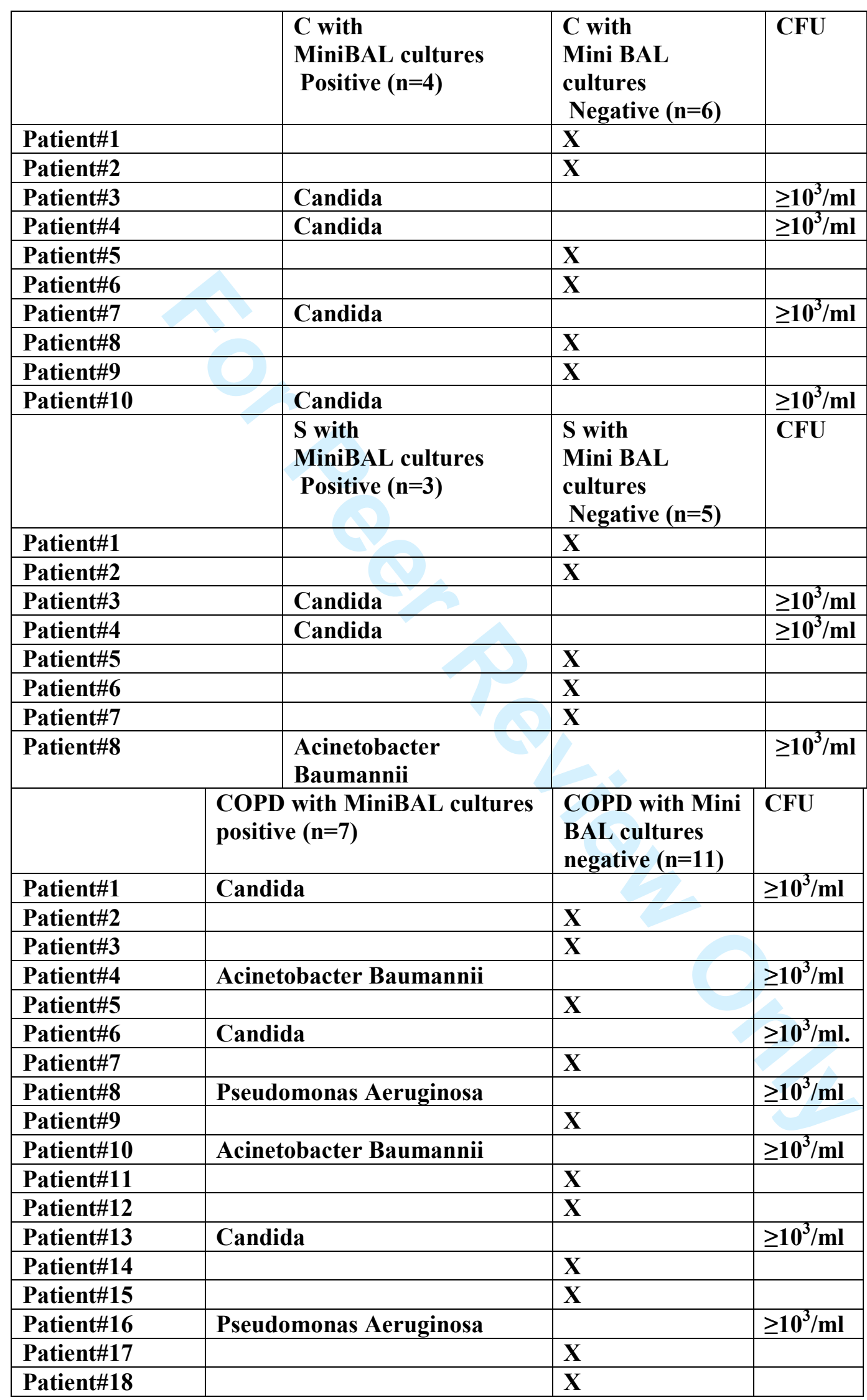




\section{Figure 1}

TLR4 expression in miniBAL and in Peripheral blood

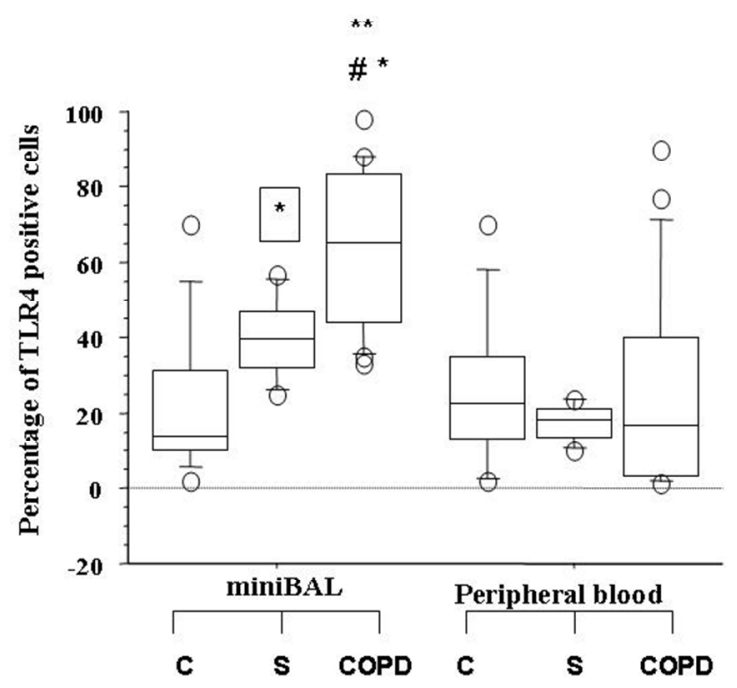

$254 \times 190 \mathrm{~mm}(96 \times 96$ DPI) 
Figure 2

IL-8 Concentrations in miniBAL and in Peripheral blood

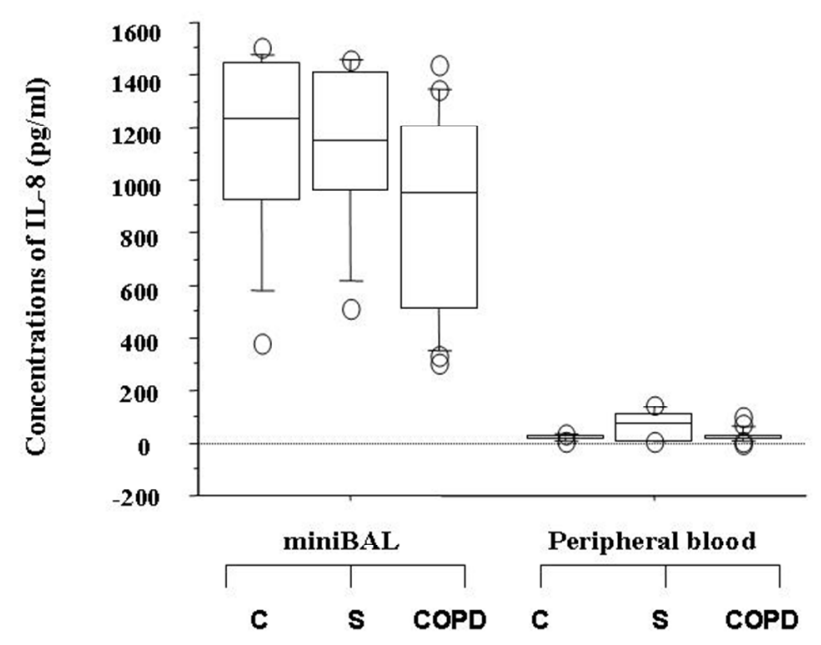

$254 \times 190 \mathrm{~mm}(96 \times 96 \mathrm{DPI})$ 


\section{Figure 3}

IP-10 Concentrations in miniBAL and in Peripheral blood

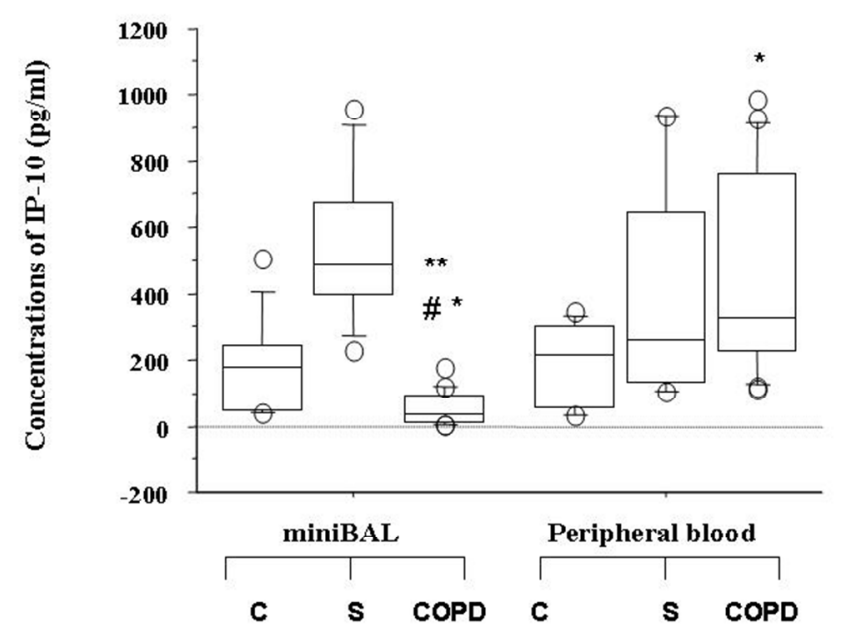

$254 \times 190 \mathrm{~mm}(96 \times 96$ DPI $)$ 


\section{Figure 4}

Foxp3 expression in miniBAL and in Peripheral blood

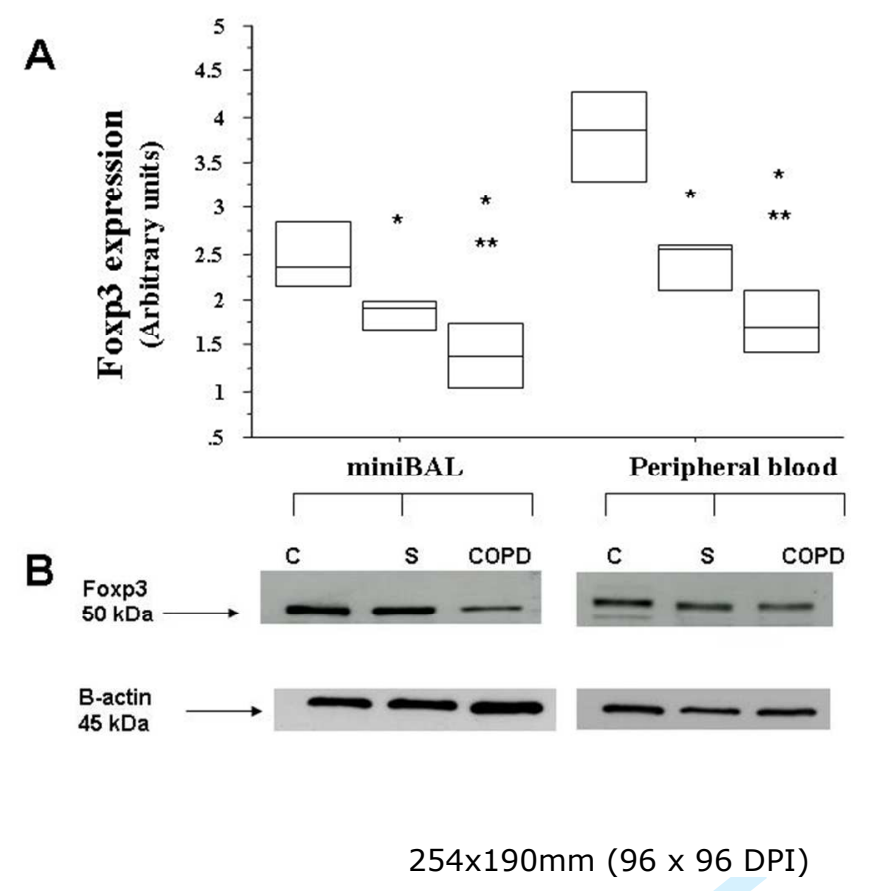




\section{Figure 5}

\section{TLR4 expression in miniBAL correlates with} CPIS score

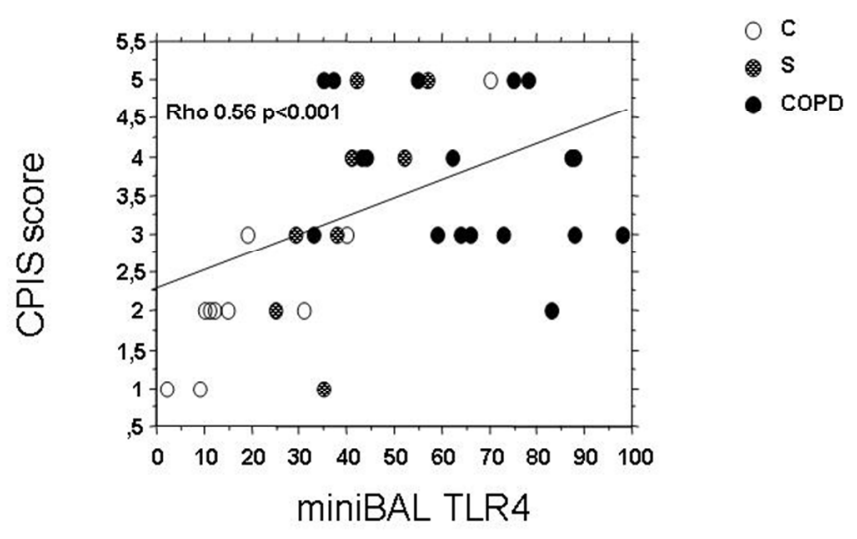

$254 \times 190 \mathrm{~mm}(96 \times 96$ DPI) 